\title{
Investigation of an Abbreviated 4 and 8 Item Version of the PTSD Checklist 5
}

*For correspondence:

Matthew.Price@uvm.edu (MP)

Present address: 2 Colchester Ave, Burlington VT 05405

\section{Matthew Price ${ }^{1 *}$, Derek D. Szafranski ${ }^{2}$, Katherine van Stolk-Cooke ${ }^{1}$, Daniel F. Gros $^{2,3}$}

${ }^{1}$ Center for Research on Emotion, Stress, and Technology, Department of Psychological Science, University of Vermont; ${ }^{2}$ Mental Health Service, Ralph H. Johnson Veterans Affairs Medical Center, Charleston, SC, USA; ${ }^{3}$ Department of Psychiatry and Behavioral Sciences, Medical University of South Carolina, Charleston, SC, USA

Abstract Posttraumatic stress disorder (PTSD) is a significant public health concern associated with marked impairment across the lifespan. Exposure to traumatic events alone, however, is insufficient to determine if an individual has PTSD. PTSD is a heterogeneous diagnosis such that assessment of all 20 symptoms is problematic in time-limited treatment settings. Brief assessment tools that identify those at risk for PTSD and measure symptom severity are needed to improve access to care and assess treatment response. The present study evaluated abbreviated measures of PTSD symptoms derived from the PTSD Checklist for DSM-5 (PCL-5) - a 20-item validated measure of PTSD symptoms - across two studies. In the first, using a community sample of adults exposed to a traumatic event, 4- and 8-item versions of the PCL-5 were identified that were highly correlated with the full PCL-5. In the second, using a sample of combat veterans, the 4 - and 8 -item measures had comparable diagnostic utility to the total-scale PCL-5. These results provide support for an abbreviated measure of the PCL-5 as an alternative to the 20 -item total scale.

\section{Measure Availability}

Copies of the Abbreviated PCL-5 (4 and 8 item version) are found here:

https://www.crestresearch.org/research/abbreviated-pcl

\section{Introduction}

Posttraumatic stress disorder (PTSD) is a chronic condition characterized by prolonged distress after a traumatic event involving actual or threatened death, injury, or sexual violence (American Psychiatric Association, 2013). The diagnostic criteria for PTSD underwent substantial revisions in DSM-5 to include 20 symptoms across four symptom clusters. The revision to the diagnosis was guided by considerable empirical support and better captures the range of maladaptive post-trauma outcomes (Miller et al., 2014). PTSD is a major public health concern with $8.3 \%$ of 
the adult civilian population and $12 \%$ of military samples meeting criteria for the diagnosis in their lifetime (Hoge et al., 2014; Kilpatrick et al., 2013). Although the effects of exposure to a potentially traumatic event (PTE) can result in psychopathology, the majority of those exposed recover. Indeed, estimates of PTE exposure are as high as $89.7 \%$ among adults (Kilpatrick et al., 2013). Therefore, PTE exposure alone is insufficient to screen for PTSD and dedicated instruments are needed.

Brief assessments to screen for PTSD and determine symptom severity are needed to effectively guide care decisions. Primary care clinics, for example, screen for multiple conditions at once (Lang and Stein, 2002). Evidence-based treatments for PTSD recommend weekly symptom assessments (Foa et al., 2007; Resick and Schnicke, 1993). The assessment of all PTSD symptoms, however, proves burdensome when used as a screen or as a repeated measure of treatment progress. Estimates of the time required to complete such a measure have been as long as $15 \mathrm{~min}$ utes, which accounts for 1/2-to-1/3 of sessions in outpatient treatment settings (Lang and Stein, 2005). Brief measures were available for PTSD based on the Diagnostic and Statistical Manual Forth Edition (DSM-IV) diagnosis (Lang and Stein, 2005; Prins et al., 2004), but only one measure currently available for the DSM-5 has psychometric data available (LeBeau et al., 2014).

The current study aimed to develop abbreviated measures of PTSD symptoms according to DSM-5 criteria using data collected from two ongoing studies. The abbreviated measures were adapted from a validated 20-item measure of PTSD symptoms, the PTSD Checklist for DSM-5 (PCL5; Blevins et al., 2015). The PCL-5 is among the few validated measures of PTSD symptom severity according to DSM-5 criteria and demonstrated excellent psychometric properties (Price and van Stolk-Cooke, 2015). Two scale lengths were selected, 4-items and 8-items. These were selected a priori to allow for adequate representation of the four clusters that comprise the PTSD diagnosis while maintaining the measures' brevity. The present study employed the methodology used to create an abbreviated form of the DSM-IV version of the PCL (Lang and Stein, 2005). Specifically, scales were derived from items with the strongest correlation with the total scale and items with the strongest correlation with each symptom cluster. This strategy allows for a comparison of measures created by empirical-item selection (strongest item total correlation) and measures that correspond to the clinical presentation of the disorder (strongest item cluster correlation). It was hypothesized that abbreviated versions containing only four or eight items would be strongly correlated with the total scale and have comparable diagnostic utility when used as a screening instrument.

\section{Study 1}

Study 1 identified the items for an abbreviated form of the PCL-5 across two independent samples. Two samples were used to protect against overfitting, defined as increased performance of a measure due to the underestimation of error in a single sample (Babyak, 2004). That is, estimates derived in one sample will contain an estimate of the true performance of the measure and components that are unique to this sample (i.e., error). When applied to a new sample, the true performance is replicated but the unique components of the first sample are not. Although performance may decline, the performance in the second sample is more accurate.

\section{Methods}

Participants

Participants were 403 individuals exposed to a PTE. Recruitment occurred via Amazon's Mechanical Turk (MTurk), an online crowdsourcing platform that is a valid method to recruit samples to measure psychopathology (Arch et al., 2015; Bardeen and Fergus, 2014; Shapiro et al., 2013). For example, online survey methods were used to assess population prevalence of the revised DSM-5 PTSD diagnostic criteria (Kilpatrick et al., 2013). The sample was divided into two indepen- 
dent groups using a random number generator. The first sample served as the development sample and the second as the replication sample. Demographic information for each subsample is presented in Table 1.

Table 1. Demographic information for the study samples

\begin{tabular}{rllllll}
\hline & \multicolumn{2}{c}{ Sample 1 } & \multicolumn{2}{c}{ Sample 2 } & \multicolumn{2}{c}{ Sample 3 } \\
\hline & $\mathbf{N}$ & $\mathbf{\%}$ & $\mathbf{N}$ & $\mathbf{\%}$ & $\mathbf{N}$ & $\mathbf{\%}$ \\
Male Gender & 109 & 54.2 & 96 & 47.0 & 107 & 78.7 \\
\hline Ethnicity & & & & & & \\
White, Non-Hispanic & 140 & 69.7 & 146 & 72.3 & 86 & 63.2 \\
White, Hispanic & 20 & 10.0 & 8 & 4.0 & 2 & 1 \\
Black, Non-Hispanic & 11 & 5 & 14 & 6.9 & 44 & 32.4 \\
Black, Hispanic & 3 & 1 & 1 & 0 & 0 & 0 \\
Asian & 17 & 8 & 14 & 6.9 & 0 & 0 \\
Other & 10 & 5.0 & 33 & 16.4 & 2 & 1 \\
Fulltime Employment & 102 & 50.7 & 121 & 59.9 & 49 & 36.0 \\
PTSD Diagnosis & - & - & - & - & 41 & 30.1 \\
\hline & $\mathbf{M}$ & SD & $\mathbf{M}$ & SD & $\mathbf{M}$ & SD \\
Age & 32.92 & 11.04 & 34.8 & 10.42 & 50.20 & 14.91 \\
PCL-5 Total & 18.55 & 18.59 & 19.84 & 17.21 & 48.83 & 18.71 \\
PCL-5 4-item & 4.05 & 4.11 & 4.27 & 3.83 & 9.99 & 4.48 \\
PCL-5 8-item & 8.46 & 8.12 & 9.00 & 7.56 & 20.84 & 8.17 \\
\hline
\end{tabular}

Measures

Life Events Checklist-5 (LEC-5; Weathers et al., 2013): The LEC-5 is a 17 item self-report measure that assesses exposure to PTE across the lifespan. Participants are asked to endorse exposure to 16 known events with an additional item to assess exposure to other extraordinarily stressful events. Participants rated if they experienced the event personally, witnessed it, learned about it, experienced it as part of their job, were unsure if they experienced the event, or did not experience the event. The extended version of the LEC was administered in which participants further described the worst event. The description of the event was typed into a textbox. Participants who did not provide adequate information about their event were excluded.

PTSD Checklist-5 (PCL-5; Weathers et al., 2013): The PCL-5 is a 20-item self-report measure that assesses PTSD symptoms experienced in the last month according to DSM-5 criteria. Items assess symptoms across the four symptom clusters of PTSD (re-experiencing, dysphoria, avoidance, and hyperarousal) on a 0 to 4 Likert scale. Total scores range from 0-80, with a score of 38 or greater indicating likely PTSD (Weathers et al., 2013). Internal consistency for the whole sample was excellent, $=0.96$.

\section{Procedure}

Participants were recruited through MTurk, an online labor marketplace through which workers can be contracted to perform computer-based tasks that require human intelligence (e.g. beta testing or self-report assessments). A Human Intelligence Task (HIT) was posted to MTurk seeking participants to complete questionnaires assessing the impact of stressful events under the keywords: survey, stress, gender, women/men, and health. Participants could access the HIT 
if they were on an IP address located in the U.S. and had a 75\% prior HIT success rate. Participants were given 1 hour to complete all measures. Within each HIT, a participant was first asked to confirm their gender to obtain a gender-balanced sample. All participants completed the Life Events Checklists (LEC), and those who did not endorse a potentially traumatic event were excluded. The results are reported in accordance with the Standards for Reporting Diagnostic Accuracy statement (STARD; Bossuyt et al., 2015).

A total of 420 individuals accessed the HIT, endorsed at least one event on the LEC-5 and completed all items on the PCL-5. The validity of responses was assessed through several methods that were consistent with best-practice recommendations (Chandler et al., 2014). First, five additional questions were included that required participants to provide a specific response (e.g., For this item, please select "Extremely"). These additional items were embedded within the standardized measures to ensure participants attended to the surveys. A valid case was identified as those who answered the majority $(>3$ ) of validity questions and spent more than five minutes completing all measures. Second, the descriptions of all traumatic events written on the LEC- 5 were reviewed by a licensed clinical psychologist and three trained bachelor's level research assistants to determine if they were consistent with Criterion A for the DSM-5 diagnosis of PTSD. Agreement across the raters was 100\%. These procedures excluded 17 (4.0\%) cases. Excluded cases involved events that included the loss of a loved one in a manner that was not unexpected or violent and stressful events that were not associated with the threat of injury or death. A final sample of $N=$ 403 participants was included in Study 1.

\section{Data Analysis}

A six stage procedure was used to identify items for an abbreviated PCL that was comparable to those used in Lang and Stein (2005). The initial sample was divided into two samples of $\mathrm{n}=202$ and $\mathrm{n}=201$ using a random number generator. Four candidate abbreviated scales were evaluated: (1) a 4-item scale derived from the items with the highest corrected-item total correlation (defined as a correlation between the item and the total score with the specific item removed); (2) an 8-item scale derived from the items with the highest corrected-item total correlation; (3) a 4-item scale derived from the items with the highest corrected-item cluster correlation; and (4) an 8-item scale derived from the items with the highest corrected-item cluster correlation in Sample 1. Item selection was done (1) using items with the strongest relation to the total scale irrespective of cluster membership and (2) using items with the strongest relation to a given cluster. A corrected-item cluster correlation was calculated for items within each PTSD symptom cluster (reexperiencing, avoidance, dysphoria, hyperarousal). Each form was then correlated with the PCL-5 total score in the first and second samples. The 4- and 8-item scales with the highest correlation in both samples were retained for further evaluation.

\section{Results}

Corrected-item total correlations for the PCL-5 and each abbreviated form across the two samples are presented in Table 2 . Within sample 1, the corrected-item cluster correlation for items 19 , difficulty concentrating $(r=0.735)$, and 20 , difficulty sleeping $(r=0.738)$, were nearly identical. Versions of the 8-item scale derived from corrected-item cluster correlations with items $19(r=$ $0.973)$ and $20(r=0.965)$ were evaluated. For sample 1 , item 19 was included in the abbreviated measure because of the higher correlation.

Item total derived scales. The 4-item scale derived from the corrected-item total correlation in sample 1 accounted for $90.8 \%(r=0.953)$ of the PCL-5 variance in sample 1 and $86.9 \%(r$ 
Table 2. Corrected-item total and item cluster correlations for each item.

Note: Bolded items in the Item total column were included in the abbreviated measure derived from item total correlations. Bolded items in the Item cluster column were included in the abbreviated measure derived from item cluster correlations. Items selected for the 4-item measure used in Study 2 were B1, C2, D2, E4. Items selected for the 8-item measure were B1, B4, C1, C2, D2, D5, E4, E5

\begin{tabular}{lll}
\hline Item & Item Total & Item Cluster \\
B1: Intrusive thoughts & 0.776 & 0.819 \\
B2: Nightmares & 0.742 & 0.759 \\
B3: Reliving trauma & 0.760 & 0.749 \\
B4: Emotional cue reactivity & 0.806 & 0.788 \\
B5: Physiological cue reactivity & 0.712 & 0.700 \\
C1: Avoidance of thoughts and emotions & 0.724 & 0.774 \\
C2: Avoidance of external reminders & 0.752 & 0.774 \\
D1: Trauma-related amnesia & 0.617 & 0.609 \\
D2: Negative expectations of self, world & 0.791 & 0.810 \\
D3: Blame of self or others for trauma & 0.647 & 0.620 \\
D4: Pervasive negative emotional state & 0.823 & 0.780 \\
D5: Loss of interest & 0.810 & 0.798 \\
D6: Feeling detached & 0.723 & 0.727 \\
D7: Lack of positive emotions & 0.775 & 0.791 \\
E1: Irritability/anger & 0.757 & 0.651 \\
E2: Recklessness & 0.73 & 0.96 \\
E3: Overly alert & 0.83 & 0.615 \\
E4: Easily startled & 0.692 & 0.759 \\
E5: Difficulty concentrating & 0.736 & 0.735 \\
E6: Sleep problems & 0.749 & 0.738 \\
\hline
\end{tabular}

$=0.932)$ of the PCL-5 variance in sample 2 . The 8-item scale derived from the corrected-item total correlation in sample 1 accounted for $95.1 \%(r=0.975)$ in PCL-5 variance in sample 1 and $94.1 \%(r$ $=0.970)$ of PCL-5 variance in sample 2 .

Item cluster derived scales. The 4-item scale derived from the corrected-item cluster correlation in sample 1 accounted for $90.1 \%(r=0.949)$ of the PCL-5 variance in sample 1 and 89.3\% ( $r=0.945)$ of the PCL-5 variance in sample 2 . The 8-item scale derived from the correcteditem cluster correlation in sample 1 accounted for $95.6 \%(r=0.98)$ in PCL-5 variance in sample 1 and $94.7 \%(r=0.97)$ of PCL-5 variance in sample 2 .

Although all of the abbreviated scales were highly correlated with the total scale, the 4item cluster-derived scale and the 8-item cluster-derived scale had the strongest relations with the total scale in both samples. Internal consistency for the 4-item scale in the full sample was good, $=0.83$. Internal consistency for the 8-item scale in the full sample was excellent, $=0.91$. The items comprising each scale are provided in the note for Table 2.

\section{Discussion}

The results demonstrate that 4- and 8-item scales comprised of items from the PCL-5 were highly correlated with the total scale. The strength of these correlations was replicated across two independent samples. Internal consistency was also good for both measures. The utility of these measures as a diagnostic screener was not evaluated and an additional study was conducted with this as the primary aim. 


\section{Study 2}

The aim of Study 2 was to further evaluate the 4- and 8-item abbreviated PCL forms in a previously collected and separate sample in which diagnostic data were available. The 4- and 8-item cluster-derived scales were selected due to their performance in Study 1.

\section{Methods}

\section{Participants}

133 patients were recruited by clinic staff of a specialized evidence-based psychotherapy clinic within a large Southeastern Veterans Affairs Medical Centers (VAMC). All participants were referred to this clinic by their existing healthcare providers to receive psychotherapy. Demographic information is provided in Table 1. According to the Mini International Neuropsychiatric Interview Seventh Edition (MINI-7), 41 (30.1\%) of the sample had a primary diagnosis of PTSD, 31 (22.8\%) had a primary diagnosis of depression, $2(1.5 \%)$ had a primary diagnosis of obsessive-compulsive disorder, 7 (5.1\%) had a primary diagnosis of social anxiety disorder, and $9(6.6 \%)$ had a primary diagnosis of generalized anxiety disorder. Comorbidity was common, with 50 (36.8\%) participants meeting criteria for 2 disorders and 32 (23.5\%) participants meeting criteria for 3 disorders. These rates of comorbidity are consistent with prior work on treatment-seeking samples of combat veterans (Conner et al., 2013).

\section{Measures}

Mini International Neuropsychiatric Interview 7: The MINI-7 is a structured diagnostic interview designed to provide a brief, but accurate, assessment of a wide range of DSM-5 psychiatric disorders (American Psychiatric Association, 2013), including mood, anxiety, trauma, and substance use psychopathology. The MINI for DSM-IV has demonstrated adequate inter-rater reliability, test-retest reliability, and good inter-rater reliability with SCID diagnoses ( = 0.7; Sheehan et al., 1998). At the time of this study the MINI-7 was under review, but was expected to function comparably to the previous version. The MINI-7 served as the reference standard for the current study.

PTSD Checklist-5: The PCL-5 was used. Internal consistency for the sample was excellent, $=0.94$.

\section{Procedures}

All procedures were approved by the local VAMC Research and Development committee as well as the Institutional Review Board at the affiliated university. Upon referral for psychotherapy, all participants' medical records were reviewed for appropriateness of the services rendered (e.g., diagnosis of psychopathology). Participants were scheduled for an intake evaluation and administered the MINI-7 to assess/confirm the presence of a disorder based on DSM-5 diagnostic criteria. Only those with a psychotic spectrum disorder diagnosis or who were acutely suicidal were excluded. All participants were assigned to either a licensed psychologist, unlicensed psychologist, or doctoral student under the supervision of a licensed psychologist for all assessments. As part of the initial intake session(s) all participants completed the questionnaire battery, including the PCL-5. 


\section{Data Analysis}

Correlations between each abbreviated form and total score PCL-5 were obtained. The diagnostic utility of the abbreviated forms and the total PCL-5 were evaluated using receiver operating characteristic (ROC) curves. Diagnoses obtained from the MINI-7 were used as the dependent variable. Area under the curve (AUC) and sensitivity/specificity for each form were obtained. An AUC greater than 0.70 is adequate with higher values indicating improved accuracy of the measure (Hanley and McNeil, 1982). Positive predictive values (PPVs) and negative predictive values (NPVs) were also calculated. PPVs and NPVs correspond to the proportion of true positives and true negatives correctly categorized by a measure. Values closer to $100 \%$ suggest the measure has greater statistical accuracy. Specific cutoff criteria were not specified a priori because of limited information as to the performance of the current scales.

\section{Results}

Internal consistency for both abbreviated measures was comparable to that obtained in Study 1 (4-item: $=0.82 ; 8$-item: $=0.90)$. The 4 -item abbreviated measure accounted for $86 \%(r=$ 0.93) of the variance in the total PCL-5. The 8-item abbreviated measure accounted for $94.1 \%(r=$ 0.97) of the variance in the total PCL-5.

According to the MINI-7, 30.1\% $(n=41)$ of the sample met diagnostic criteria for PTSD using DSM-5 criteria. ANOVAs indicated that the PCL total scores (PTSD: $M=58.98, S D=14.58$; No PTSD: $M=44.30, S D=18.64)$ differed among those with and without a diagnosis, $F(1,131=19.93$, $p<.001$ ). There was a significant difference between these groups on the 8-item measure (PTSD: $M=24.93, S D=6.61 ;$ No PTSD: $M=19.02, S D=8.17$ ) and 4-item measure (PTSD: $M=12.32, S D=$ 3.58; No PTSD: $M=8.95, S D=4.46)$ as well, 8-item: $F(1,131=16.57, p<.001$, 4-item: $F(1,131=$ $18.17, \mathrm{p}<.001$.

The AUC for the ROC curve using the total scale for the PCL-5 was $=0.72$ ( $95 \% \mathrm{Cl}: 0.63$ to 0.82). The recommended cut score of 38 corresponded to a sensitivity of 0.90 ( $95 \% \mathrm{Cl}: 0.76$ to 0.97 ) and a specificity of 0.35 ( $95 \% \mathrm{Cl}: 0.25$ to 0.46$)$. This cut score resulted in a positive predictive value (PPV) of $38.1 \%$ and a negative predictive value (NPV) of $88.9 \%$. Additional information regarding the performance of the PCL-5 is presented in Table 3. An ROC curve using the 4-item abbreviated scale obtained an AUC $=0.72$ ( $95 \% \mathrm{Cl}: 0.62$ to 0.81 ). Cut scores for the abbreviated scales were selected to maximize sensitivity while maintaining adequate specificity. Sensitivity was prioritized to allow for a more inclusive screening instrument. A cut score of 10 corresponded to a sensitivity of 0.76 ( $95 \% \mathrm{Cl}: 0.59$ to 0.87 ) and a specificity of 0.52 ( $95 \% \mathrm{Cl}: 0.42$ to 0.63 ). This cut score resulted in a PPV of $42.0 \%$ and a NPV of $83.3 \%$. An ROC curve using the 8 -item abbreviated scale obtained an $\mathrm{AUC}=0.71$ ( $95 \% \mathrm{Cl}: 0.61$ to 0.80 ). A cut score of 19 corresponded to a sensitivity of $0.83(95 \% \mathrm{Cl}$ : 0.67 to 0.92 ) and a specificity of 0.39 ( $95 \% \mathrm{Cl}: 0.29$ to 0.50 ). This cut score resulted in a PPV of $37.8 \%$ and a NPV of 83.7\%. Sensitivity, specificity, PPV, and NPV values are provided in Table 4 and Table 5 for the 4- and 8-item scales respectively. A McNemar test compared the sensitivity and specificity of the abbreviated PCL scales to the total scale across those with and without a PTSD diagnosis (Table 6). There was no significant difference in the sensitivity of the total scale and the 4-item scale (McNemar $2(1)=3.13, p=0.07)$ or the 8-item scale $(\operatorname{McNemar} 2(1)=1.33, p=0.25)$. There was no significant difference in the specificity of the total scale and the 8-item scale (McNemar 2 $(1)=1.13, p=0.29$ ). There was a significant difference in the specificity of the total scale and the 4 -item scale such that the 4 -item scale performed better $(\operatorname{McNemar} 2(1)=10.23, p<0.01)$. Taken together, these results suggest that the abbreviated scales perform as well or better than the total scale. 
Table 3. Sensitivity and Specificity for total PCL 5 scale.

Note: Values less than 30 or greater than 65 did not provide statistics that were appreciably different were not included. Increments of 5 are presented due to space limitations. Additional information is available upon request from the contact author.

\begin{tabular}{rllll}
\hline Cutscore & Sensitivity & Specificity & PPV & NPV \\
\hline 30 & 1.00 & 0.22 & 36.3 & 100.0 \\
35 & 0.93 & 0.29 & 36.9 & 90.0 \\
38 & 0.90 & 0.35 & 38.1 & 88.9 \\
40 & 0.90 & 0.36 & 38.5 & 89.2 \\
45 & 0.78 & 0.44 & 38.1 & 81.6 \\
50 & 0.73 & 0.62 & 46.2 & 83.8 \\
54 & 0.66 & 0.70 & 49.1 & 82.1 \\
60 & 0.56 & 0.76 & 51.1 & 79.5 \\
65 & 0.42 & 0.84 & 53.1 & 76.2 \\
\hline
\end{tabular}

Table 4. Sensitivity and Specificity for 4-item abbreviated PCL 5 scale. Note: Values less than 5 or greater than 15 did not provide statistics that were appreciably different from 5 or 15 and were not included.

\begin{tabular}{rllll}
\hline Cutscore & Sensitivity & Specificity & PPV & NPV \\
\hline 5 & 0.98 & 0.15 & 34.1 & 93.8 \\
6 & 0.98 & 0.23 & 36.4 & 95.7 \\
7 & 0.93 & 0.29 & 37.3 & 90.3 \\
8 & 0.88 & 0.34 & 37.5 & 86.5 \\
9 & 0.85 & 0.39 & 38.9 & 86.0 \\
10 & 0.76 & 0.52 & 42.0 & 83.3 \\
11 & 0.68 & 0.62 & 44.4 & 81.4 \\
12 & 0.59 & 0.72 & 48.0 & 79.5 \\
13 & 0.51 & 0.79 & 52.5 & 78.5 \\
14 & 0.46 & 0.83 & 54.3 & 77.8 \\
15 & 0.37 & 0.87 & 55.6 & 75.5 \\
\hline
\end{tabular}

\section{Discussion}

Results from this study provide additional support for the strength of the abbreviated measures. Both scales were highly correlated with the total scale and demonstrated good internal consistency. The diagnostic utility of the measures was comparable to that of the total scale, which suggests that they may be useful as abbreviated screening tools. Interestingly, the 4-item scale had an AUC that was nearly identical to that of the total scale, which suggests that this may be a better screening measure for PTSD.

\section{General Discussion}

The results of the present investigation provide support for the utility of abbreviated PTSD scales to assess diagnostic status and symptom severity. The two scales, which were derived from a psychometrically validated PTSD measure, were highly correlated with the total scale across three independent samples recruited from the community and an outpatient veteran's clinic. The replication of the strong relations suggests that these measures are robust and not unique to a single sample. Furthermore, the items selected for the current abbreviated measures map onto the majority of the most commonly endorsed symptoms by combat soldiers (Hoge et al., 2014). This 
Table 5. Sensitivity and specificity for 8-item abbreviated PCL 5 scale.

Note: Values less than 13 or greater than 35 did not provide statistics that were appreciably different from 13 or 35 and were not included. No participants had scores of 33 or 34 and thus these rows were omitted.

\begin{tabular}{rllll}
\hline Cutscore & Sensitivity & Specificity & PPV & NPV \\
\hline 13 & 0.95 & 0.23 & 35.4 & 91.3 \\
14 & 0.93 & 0.26 & 35.8 & 88.9 \\
15 & 0.93 & 0.27 & 36.2 & 89.3 \\
16 & 0.88 & 0.29 & 35.6 & 84.4 \\
17 & 0.83 & 0.35 & 36.2 & 82.1 \\
18 & 0.83 & 0.38 & 37.4 & 83.3 \\
19 & 0.83 & 0.39 & 37.8 & 83.7 \\
20 & 0.78 & 0.47 & 39.5 & 82.7 \\
21 & 0.76 & 0.55 & 43.1 & 83.6 \\
22 & 0.68 & 0.61 & 43.8 & 81.2 \\
23 & 0.66 & 0.65 & 45.8 & 81.1 \\
24 & 0.59 & 0.71 & 47.1 & 79.3 \\
25 & 0.54 & 0.72 & 45.8 & 77.6 \\
26 & 0.54 & 0.78 & 52.4 & 79.1 \\
27 & 0.49 & 0.80 & 52.6 & 77.8 \\
28 & 0.46 & 0.85 & 57.6 & 78.0 \\
29 & 0.39 & 0.87 & 57.1 & 76.2 \\
30 & 0.32 & 0.88 & 54.2 & 74.3 \\
31 & 0.20 & 0.94 & 57.1 & 72.3 \\
32 & 0.17 & 0.96 & 63.6 & 72.1 \\
35 & 0.05 & 0.99 & 66.7 & 70.0 \\
\hline & & & & \\
\hline
\end{tabular}

suggests that the techniques used in the current studies identified a set of items that may generalize to other trauma-exposed samples. Taken together, these findings further suggest that 4- and 8-item versions of the full 20 item PCL measure may ease time restraints for clinical assessment and symptom monitoring.

Selection of either abbreviated form or the total form should depend on the specific needs of the assessor. The 8-item scale had a stronger correlation with the total scale, greater internal consistency, and allowed for more variability in patient response. The 4-item scale demonstrated better specificity and comparable sensitivity with a cut score of 10 . These features suggest that the 4-item scale may be a stronger screening instrument when using a cut score of 10 whereas the 8item scale may prove better at monitoring treatment progress because it allows for more variability in responses. However, these recommendations are not strict guidelines and should be empirically evaluated. The cut scores selected for the current study maximized sensitivity while maintaining adequate specificity for use as a screening instrument. That is, they perform better identifying those at risk who are highly unlikely to have PTSD and those who warrant further assessment for PTSD by trained professionals. As such the selected cut scores were suited for population-based or clinic-wide evaluations. The sensitivity and specificity are provided for a range of cut scores, however, to allow clinicians and researchers to choose an optimal score for their needs.

The validation of these brief PTSD measures has several implications. The abbreviated forms of the PCL-5 could allow for a more efficient assessment of PTSD symptoms. Such efficiency particularly valuable because outpatient mental health clinics and primary care settings are required to screen regularly for multiple mental health conditions (Lang and Stein, 2005). The availability of brief assessment tools often determines if a condition will be assessed. For example, a 
Table 6. Cross tabulation of 4-item, 8-item PCL, and total PCL and diagnostic status according to the MINI-7. Note: ${ }^{1}=$ diagnosis made at a score of $10 .{ }^{2}=$ diagnosis made at a score of $19 .{ }^{3}=$ Diagnosis made at a score of 38 .

\begin{tabular}{|c|c|c|c|}
\hline \multicolumn{3}{|c|}{ MINI-7 Diagnosis } & \multirow[t]{2}{*}{ Total } \\
\hline 4-item diagnosis ${ }^{1}$ & No Diagnosis & PTSD & \\
\hline No Diagnosis & 48 & 10 & 58 \\
\hline PTSD & 44 & 31 & 75 \\
\hline \multicolumn{4}{|l|}{ 8-item diagnosis ${ }^{2}$} \\
\hline No Diagnosis & 36 & 7 & 43 \\
\hline PTSD & 56 & 34 & 90 \\
\hline \multicolumn{4}{|l|}{ PCL diagnosis ${ }^{3}$} \\
\hline No Diagnosis & 32 & 4 & 36 \\
\hline PTSD & 60 & 37 & 97 \\
\hline
\end{tabular}

2-item screening instrument for depression has greatly increased the frequency with which the condition is assessed (Löwe et al., 2005). Indeed, brief measures for depression and PTSD were developed for use within the VAMCs; however, the accuracy and acceptability of these measures have been questioned (Seal et al., 2008). Given the high prevalence of trauma exposure among adults and the significant negative impact of PTSD on quality of life (Pagotto et al., 2015), these 4- or 8-item measures of PTSD symptoms have the potential to improve public health services by identifying those who would benefit from treatment. Screen-to-treat methods are especially important given evidence that victims of traumatic events such as physical injury (Bryant et al., 2010) and sexual assault (Price et al., 2014) are unlikely to seek treatment for PTSD.

Treatment and research protocols that call for repeated assessment of PTSD symptoms would benefit from the abbreviated forms as well. Two of the most well validated treatments for PTSD (Monson et al., 2006; Powers et al., 2010), prolonged exposure (Foa et al., 2007) and cognitive processing therapy (Resick and Schnicke, 1993) require weekly symptom assessments. Abbreviated measures that provide severity data to the clinician will maintain protocol fidelity and save valuable session time. Similarly, research protocols that require participants to complete daily assessments of PTSD symptoms, such as in ecological momentary assessment paradigms (Shiffman et al., 2008), would benefit from the availability of an abbreviated form to reduce user burden (Dedert et al., 2014; Kleim et al., 2013). Indeed, an advantage of the scales proposed in the current study is the use of continuous scores as opposed to a dichotomous outcome (diagnosis or no diagnosis). Prior to conducting such studies, however, it is necessary to determine if these abbreviated measures provide reliable and valid indices of change.

Another brief measure, the National Stressful Events Survey for PTSD-Short Scale (NSESSSPTSD; LeBeau et al., 2014), was developed recently to provide a continuous measure of PTSD symptoms. The measures proposed in this study and the NSESSS-PTSD performed well in their respective studies. The NSESSS-PTSD has not yet been evaluated in a clinical sample and data as to its diagnostic utility are not yet available, however. Thus, further work is required before firm conclusions can be drawn as to which measure in optimal. Interestingly, the items selected in the present studies and those in the NSESSS-PTSD are similar, which suggests that there may be a core set of symptoms that identify those with PTSD. Further evaluation of these symptoms would allow for a harmonization among these measures to create a gold standard instrument.

The present study had several limitations. First, the sample in Study 1 did not include the diagnostic status of participants. The lack of such information limits the strength of the conclusions that can be drawn about the diagnostic utility of the measure as a screener. Similarly, the sample 
sizes for the current study, while comparable to prior studies (Lang and Stein, 2005; Prins et al., 2004), were modest for psychometric studies. The replication of these findings in larger samples with full diagnostic information is needed to improve confidence in these abbreviated forms. The sample used in Study 1 was a heterogeneous trauma sample and it is thus unclear if the measures function differently in specific subsamples (e.g., sexual assault victims or victims of an accident). Alternatively, the sample used in Study 2 consisted entirely of veterans, which may have influenced the results. Additional population-specific evaluations are warranted. Although diagnoses were verified by licensed clinical psychologists, reliability data was not available for the sample in Study 2. The current study did not use the Clinician Administered PTSD Scale (CAPS), which is a gold standard diagnostic instrument for PTSD. The current study was unable to evaluate other forms of validity that increase confidence in psychometrically sound measures. Additional investigations are needed to confirm that these abbreviated scales have comparable content, criterion, and construct validity. The psychometric properties of the PCL-5 as a diagnostic measure in Study 2 were limited and should be evaluated further in a larger sample. An initial study on the PCL-5 factor structure suggested that it mirrors those used in the DSM-5 (Blevins et al., 2015), but further work examining its use as a diagnostic instrument is needed.

The present study supports the use of abbreviated measures of PTSD symptoms. The 4-item measure is recommended as a screening instrument, whereas the 8-item measure is recommended for monitoring symptoms. Additional brief measures are under evaluation, such as the PC-PTSD-5 (NCPTSD, 2015) and NSESSS-PTSD (LeBeau et al., 2014), to provide clinicians and researchers with a range of tools to assess and diagnose PTSD. It is strongly recommended that other research teams evaluate the performance of these measures in independent samples to further evaluate their utility. The ability to derive these scales from a widely used measure of PTSD is a benefit of the proposed scales. Further evaluation of these measures, therefore, can occur as secondary analyses. These abbreviated measures will facilitate the efficient identification of those who would benefit from trauma-focused treatment and enable symptom monitoring with minimal burden to the patient.

\section{References}

Afzali, M. H., Sunderland, M., Teesson, M., Carragher, N., Mills, K., and Slade, T. (2017). A network approach to the comorbidity between posttraumatic stress disorder and major depressive disorder: The role of overlapping symptoms. Journal of Affective Disorders, 208:490-496.

Alcántara, C., Casement, M. D., and Lewis-Fernández, R. (2013). Conditional risk for PTSD among Latinos: A systematic review of racial/ethnic differences and sociocultural explanations. Clinical Psychology Review, 33(1):107-119.

Armour, C., Fried, E. I., Deserno, M. K., Tsai, J., and Pietrzak, R. H. (2017). A network analysis of dsm-5 posttraumatic stress disorder symptoms and correlates in us military veterans. Journal of anxiety disorders, 45:49-59.

Babcock, J. C., Roseman, A., Green, C. E., and Ross, J. M. (2008). Intimate partner abuse and PTSD symptomatology: Examining mediators and moderators of the abuse-trauma link. Journal of Family Psychology, 22(6):809818. Place: US Publisher: American Psychological Association.

Bernstein, D. P., Fink, L., Handelsman, L., and Foote, J. (1998). Childhood trauma questionnaire. Assessment of family violence: $A$ handbook for researchers and practitioners.

Borsboom, D. and Cramer, A. O. (2013). Network Analysis: An Integrative Approach to the Structure of Psychopathology. Annual Review of Clinical Psychology, 9(1):91-121. _eprint: https://doi.org/10.1146/annurevclinpsy-050212-185608.

Cero, I. and Kilpatrick, D. G. (2020). Network Analysis of Posttraumatic Stress Disorder Symptoms in a National Sample of U.S. Adults: Implications for the Phenotype and the ICD-11 Model of PTSD. Journal of Traumatic Stress, 33(1):52-63. _eprint: https://onlinelibrary.wiley.com/doi/pdf/10.1002/jts.22481. 
Cloitre, M., Stolbach, B. C., Herman, J. L., Kolk, B. v. d., Pynoos, R., Wang, J., and Petkova, E. (2009). A developmental approach to complex PTSD: Childhood and adult cumulative trauma as predictors of symptom complexity. Journal of Traumatic Stress, 22(5):399-408. _eprint: https://onlinelibrary.wiley.com/doi/pdf/10.1002/jts.20444.

Cloitre, M., Stovall-McClough, K. C., Nooner, K., Zorbas, P., Cherry, S., Jackson, C. L., Gan, W., and Petkova, E. (2010). Treatment for PTSD Related to Childhood Abuse: A Randomized Controlled Trial. American Journal of Psychiatry, 167(8):915-924. Publisher: American Psychiatric Publishing.

Contractor, A. A., Greene, T., Dolan, M., Weiss, N. H., and Armour, C. (2020). Relation between PTSD symptom clusters and positive memory characteristics: A network perspective. Journal of Anxiety Disorders, 69:102157.

Epskamp, S., Borsboom, D., and Fried, E. I. (2018). Estimating psychological networks and their accuracy: A tutorial paper. Behavior Research Methods, 50(1):195-212.

Fierros, M. and Smith, C. (2006). The Relevance of Hispanic Culture to the Treatment of a Patient with Posttraumatic Stress Disorder (PTSD). Psychiatry (Edgmont), 3(10):49-56.

Galatzer-Levy, I. R. and Bryant, R. A. (2013). 636,120 ways to have posttraumatic stress disorder. Perspectives on Psychological Science, 8(6):651-662.

Gonzalez, F. R., Benuto, L. T., and Casas, J. B. (2020). Prevalence of Interpersonal Violence Among Latinas: A Systematic Review. Trauma, Violence, \& Abuse, 21(5):977-990. Publisher: SAGE Publications.

Grassi-Oliveira, R. and Stein, L. M. (2008). Childhood maltreatment associated with PTSD and emotional distress in low-income adults: The burden of neglect. Child Abuse \& Neglect, 32(12):1089-1094.

Grasso, D. J., Felton, J. W., and Reid-Quiñones, K. (2015). The Structured Trauma-Related Experiences and Symptoms Screener (STRESS): Development and Preliminary Psychometrics. Child Maltreatment, 20(3):214220. Publisher: SAGE Publications Inc.

Hall-Clark, B., Sawyer, B., Golik, A., and Asnaani, A. (2016). Racial/Ethnic Differences in Symptoms of Posttraumatic Stress Disorder. Current Psychiatry Reviews, 12(2):124-138.

Hien, D. and Ruglass, L. (2009). Interpersonal partner violence and women in the United States: An overview of prevalence rates, psychiatric correlates and consequences and barriers to help seeking. International journal of law and psychiatry, 32(1):48-55.

Hoeboer, C., de Roos, C., van Son, G. E., Spinhoven, P., and Elzinga, B. (2021). The effect of parental emotional abuse on the severity and treatment of PTSD symptoms in children and adolescents. Child Abuse \& Neglect, 111:104775.

Isvoranu, A.-M., Epskamp, S., and Cheung, M. (2020). Network Models of Post-traumatic Stress Disorder: A Meta-analysis. Technical report, PsyArXiv. type: article.

Jovanovic, T., Blanding, N. Q., Norrholm, S. D., Duncan, E., Bradley, B., and Ressler, K. J. (2009). Childhood abuse is associated with increased startle reactivity in adulthood. Depression and Anxiety, 26(11):1018-1026. _eprint: https://onlinelibrary.wiley.com/doi/pdf/10.1002/da.20599.

Kaltman, S., Green, B. L., Mete, M., Shara, N., and Miranda, J. (2010). Trauma, Depression, and Comorbid PTSD/Depression in a Community Sample of Latina Immigrants. Psychological trauma: theory, research, practice and policy, 2(1):31-39.

Kilpatrick, D. G., Resnick, H. S., Milanak, M. E., Miller, M. W., Keyes, K. M., and Friedman, M. J. (2013). National Estimates of Exposure to Traumatic Events and PTSD Prevalence Using DSM-IV and DSM-5 Criteria. Journal of Traumatic Stress, 26(5):537-547. _eprint: https://onlinelibrary.wiley.com/doi/pdf/10.1002/jts.21848.

Machlin, L., Miller, A. B., Snyder, J., McLaughlin, K. A., and Sheridan, M. A. (2019). Differential associations of deprivation and threat with cognitive control and fear conditioning in early childhood. Frontiers in behavioral neuroscience, 13:80.

Marshall, G. N., Schell, T. L., and Miles, J. N. V. (2009). Ethnic Differences in Posttraumatic Distress: Hispanics' Symptoms Differ in Kind and Degree. Journal of consulting and clinical psychology, 77(6):1169-1178.

McCrory, E., De Brito, S. A., and Viding, E. (2012). The link between child abuse and psychopathology: A review of neurobiological and genetic research. Journal of the Royal Society of Medicine, 105(4):151-156. Publisher: SAGE Publications. 
McCrory, E. J., Gerin, M. I., and Viding, E. (2017). Annual research review: childhood maltreatment, latent vulnerability and the shift to preventative psychiatry-the contribution of functional brain imaging. Journal of child psychology and psychiatry, 58(4):338-357.

McLaughlin, K. A. and Sheridan, M. A. (2016). Beyond Cumulative Risk: A Dimensional Approach to Childhood Adversity. Current Directions in Psychological Science, 25(4):239-245. Publisher: SAGE Publications Inc.

McNally, R. J. (2012). The Ontology of Posttraumatic Stress Disorder: Natural Kind, Social Construction, or Causal System? Clinical Psychology: Science and Practice, 19(3):220-228. _eprint: https://onlinelibrary.wiley.com/doi/pdf/10.1111/cpsp.12001.

Norman, R. E., Byambaa, M., De, R., Butchart, A., Scott, J., and Vos, T. (2012). The Long-Term Health Consequences of Child Physical Abuse, Emotional Abuse, and Neglect: A Systematic Review and Meta-Analysis. PLOS Medicine, 9(11):e1001349. Publisher: Public Library of Science.

Ogle, C. M., Rubin, D. C., and Siegler, I. C. (2013). The impact of the developmental timing of trauma exposure on PTSD symptoms and psychosocial functioning among older adults. Developmental Psychology, 49(11):21912200. Place: US Publisher: American Psychological Association.

Pole, N., Best, S., Metzler, T., and Marmar, C. (2005). Why are Hispanics at greater risk for PTSD? Cultural diversity \& ethnic minority psychology, 11:144-61.

Price, M., Legrand, A. C., Brier, Z. M. F., and Hébert-Dufresne, L. (2019). The symptoms at the center: Examining the comorbidity of posttraumatic stress disorder, generalized anxiety disorder, and depression with network analysis. Journal of Psychiatric Research, 109:52-58.

Price, M., Szafranski, D. D., van Stolk-Cooke, K., and Gros, D. F. (2016). Investigation of abbreviated 4 and 8 item versions of the PTSD Checklist 5. Psychiatry Research, 239:124-130.

Ross, N. D., Kaminski, P. L., and Herrington, R. (2019). From childhood emotional maltreatment to depressive symptoms in adulthood: The roles of self-compassion and shame. Child Abuse \& Neglect, 92:32-42.

Scher, C. D., Forde, D. R., McQuaid, J. R., and Stein, M. B. (2004). Prevalence and demographic correlates of childhood maltreatment in an adult community sample. Child Abuse \& Neglect, 28(2):167-180.

Schumm, J. A., Briggs-Phillips, M., and Hobfoll, S. E. (2006). Cumulative interpersonal traumas and social support as risk and resiliency factors in predicting PTSD and depression among inner-city women. Journal of Traumatic Stress, 19(6):825-836. _eprint: https://onlinelibrary.wiley.com/doi/pdf/10.1002/jts.20159.

Shahar, B., Doron, G., and Szepsenwol, O. (2015). Childhood Maltreatment, Shame-Proneness and Self-Criticism in Social Anxiety Disorder: A Sequential Mediational Model. Clinical Psychology \& Psychotherapy, 22(6):570579. _eprint: https://onlinelibrary.wiley.com/doi/pdf/10.1002/cpp.1918.

Strauss, M. (1988). Manual for the Conflict Tactics Scales (CTS) | Office of Justice Programs.

Sullivan, T. P., Fehon, D. C., Andres-Hyman, R. C., Lipschitz, D. S., and Grilo, C. M. (2006a). Differential relationships of childhood abuse and neglect subtypes to PTSD symptom clusters among adolescent inpatients. Journal of Traumatic Stress, 19(2):229-239. _eprint: https://onlinelibrary.wiley.com/doi/pdf/10.1002/jts.20092.

Sullivan, T. P., Fehon, D. C., Andres-Hyman, R. C., Lipschitz, D. S., and Grilo, C. M. (2006b). Differential relationships of childhood abuse and neglect subtypes to PTSD symptom clusters among adolescent inpatients. Journal of Traumatic Stress, 19(2):229-239. _eprint: https://onlinelibrary.wiley.com/doi/pdf/10.1002/jts.20092.

Warner, L. A., Alegría, M., and Canino, G. (2012). Childhood Maltreatment Among Hispanic Women in the United States: An Examination of Subgroup Differences and Impact on Psychiatric Disorder. Child Maltreatment, 17(2):119-131. Publisher: SAGE Publications Inc.

Widom, C. S., Czaja, S. J., and Dutton, M. A. (2008). Childhood victimization and lifetime revictimization. Child Abuse \& Neglect, 32(8):785-796.

Williams, M., Printz, D. M., and DeLapp, R. C. (2018). Assessing Racial Trauma With the Trauma Symptoms of Discrimination Scale. Psychology of Violence, 8(6):735-747.

Wiseman, H., Hamilton-Giachritsis, C., and Hiller, R. M. (2021). The Relevance of Cognitive Behavioral Models of Post-Traumatic Stress Following Child Maltreatment: A Systematic Review. Trauma, Violence, \& Abuse, 22(1):191-206. Publisher: SAGE Publications.

Woods, S. J. and Wineman, N. M. (2004). Trauma, posttraumatic stress disorder symptom clusters, and physical health symptoms in postabused women. Archives of Psychiatric Nursing, 18(1):26-34. 